\title{
FOXO1 is crucial in glioblastoma cell tumorigenesis and regulates the expression of SIRT1 to suppress senescence in the brain
}

\author{
HAN YAN and ANHUA WU \\ Department of Neurosurgery, The First Affiliated Hospital of \\ China Medical University, Shenyang, Liaoning 110001, P.R. China
}

Received June 6, 2016; Accepted August 14, 2017

DOI: $10.3892 / \mathrm{mmr} .2017 .8146$

\begin{abstract}
In the present study, the role of Forkhead Box O1 (FOXO1) in glioblastoma (GBM) cell tumorigenesis was examined and the underlying mechanisms were investigated. Reverse transcription-quantitative polymerase chain reaction and western blot analyses were used to analyze the expression of FOXO1 in GBM cell lines (LN18 and T98G) and tissues. Compared with the control groups, FOXO1 was significantly downregulated in the GBM tissues and GBM cell lines $(\mathrm{P}<0.05)$. The effects of the expression of FOXO1 on GBM cell proliferation and cell cycle were examined using flow cytometry. The overexpression of FOXO1 markedly inhibited LN18 and T98G cell proliferation and arrested cell cycle at the $G_{0} / G_{1}$ phase. In addition, FOXO1 facilitated cel senescence through regulation of the expression of sirtuin 1 . Epithelial-mesenchymal transition (EMT) is a complex process, which affects cell growth, invasion and metastasis. The results of the present study revealed that FOXO1 inhibited EMT and metastasis in GBM. These finding revealed a novel mechanism of FOXO1 in the suppression of tumorigenesis and metastasis of GBM cells and suggested that FOXO1 may be a potential therapeutic target for treating GBM.

\section{Introduction}

Glioblastoma (GBM) has become the most aggressive and common type of central nervous system malignancy worldwide (1). However, the causes of the carcinogenesis and progression of GBM remain to be elucidated. Several studies have indicated that the aberrant expression of the Forkhead Box (FOX) family protein has a key function in tumor growth, metastasis and response to cancer therapy (2-6).
\end{abstract}

Correspondence to: Dr Anhua Wu, Department of Neurosurgery, The First Affiliated Hospital of China Medical University, 155 Nanjing Road, Shenyang, Liaoning 110001, P.R. China

E-mail: anhuawu1988@sina.com

Key words: Forkhead Box O1, sirtuin 1, epithelial-mesenchymal transition, metastasis, senescence
The FOXO family includes FOXO1, FOXO3, FOXO4 and FOXO6. They all have a crucial function in gene transcription, which mediates cell processes, including DNA damage repair, apoptotic cell death, glucose metabolism, cell cycle control and carcinogenesis (7). FOXO members consist of a conserved domain, which binds the DNA sequence TTGTTTAC at target gene $(8,9)$. Several reports have demonstrated that FOXOs function in autophagy (10-12). In oxidative stress, FOXO1 can promote autophagy, which may due to its tumor suppressor activity (13).

Sirtuin 1 (SIRT1) is the mammalian orthologue of Sir2. It has been reported that SIRT1 has an important function in cell senescence $(14,15)$. Nicotinamide adenine dinucleotide and nicotinamide can regulate the activity of SIRT1. The activation of SIRT1 protects cardiomyocytes from death and promotes the survival of neurons. In different tissues, SIRT1 has specific functions. For example, SIRT1 promotes gluconeogenesis and fatty acid oxidation under nutrient deprivation in the liver (16). SIRT1 is also involved in pancreatic $\beta$-cell survival and insulin secretion through interacting with the FOXO family (17) and inhibiting of uncoupling protein 2 (18), respectively.

Senescence is a state in which cells undergo specific alterations in gene expression and cellular morphology, accompanied by loss of the ability to proliferate. During cell senescence, $\beta$-galactosidase, which is associated with senescence, is activated, followed by cell cycle arrest at the $G_{0} / G_{1}$ phase and a marked increase in the expression of cyclin-dependent kinase inhibitor $(19,20)$.

In the present study, it was revealed that FOXO1 was significantly downregulated in the GBM tissues and GBM cell lines. FOXO1 inhibited cell proliferation via arresting the cell cycle at the $\mathrm{G}_{0} / \mathrm{G}_{1}$ phase. In addition, FOXO1 facilitated cell senescence through regulation of sirtuin 1 expression. In addition, FOXO1 suppressed epithelial mesenchymal transition and metastasis. These findings suggested a novel mechanism of FOXO1 in the suppression of tumorigenesis and metastasis of GBM cells and suggested that FOXO1 may be a potential therapeutic target for treating GBM.

\section{Materials and methods}

Cell culture. Human U87, T98G and LN18 GBM cell lines were purchased from the America Type Culture Collection (Manassas, VA, USA) and cultured in DMEM (Gibco; Thermo 
Fisher Scientific, Inc., Waltham, MA, USA) with 10\% FBS (Sigma-Aldrich; Merck Millipore, Darmstadt, Germany) in $5 \% \mathrm{CO}_{2}$ at $37^{\circ} \mathrm{C}$.

Plasmid constructs and transfection. The human U87, T98G and LN18 GBM cells were grown in a 6-well plate to almost $70 \%$ confluence, follow by transfection with $3 \mu \mathrm{g}$ of plasmids. The empty vector pcDNA3.1 plasmid and the FOXO1-containing plasmid 1 (pcDNA3.1-FOXO) were used (VigeneBio, Shandong, China). Transfection was performed using Lipofectamine ${ }^{\circledR} 2000$ reagent (Invitrogen; Thermo Fisher Scientific, Inc.), according to the manufacturer's protocol, for U87, T98G and LN18 transfection.

Cell cycle analysis. The cells were detatched using $0.25 \%$ trypsin and collected when the cells had reached almost $70-80 \%$ confluence, following which they were fixed with $75 \%$ ethanol overnight. $\sim 3 \times 10^{4}$ cells were treated with $1 \mathrm{mg} / \mathrm{ml}$ RNase A (Sigma-Aldrich; Merck Millipore) at $37^{\circ} \mathrm{C}$ for $40 \mathrm{~min}$, resuspended in PBS and stained with propidium iodide for $30 \mathrm{~min}$ in the dark. Finally, the DNA contents were measured using a FACScan flow cytometry system. All experiments were performed three times.

Tissue samples. The 143 pairs' human GBM tissues and adjacent normal tissues were obtained from patients at The First Affiliated Hospital of China Medical University (Shenyang, China) between 2011 and 2015. All patients were diagnosed by pathological examination as having GBM. A total of 85 were male and 58 were female patients. The present study approved by the Institutional Research Ethics Committee of The First Affiliated Hospital of China Medical University. All patients were informed and provided written informed consent. All tissue samples were collected and frozen in liquic nitrogen and stored at $-80^{\circ} \mathrm{C}$ until processing. The expression of FOXO1 in tissues was determined by reverse transcription-quantitative polymerase chain reaction (RT-qPCR). The mean value of FOXO1 content in tumor cells acted as the standard. Therefore, higher values than the standard value was denoted as high expression and lower values than the standard value was denote

MTT assay. An MTT assay was used to detect cell proliferation, as described previously (21). In brief, the cells were seeded at $3 \times 10^{3}$ per well in a 96-well plate and incubated with $20 \mu \mathrm{l}$ MTT $\left(10 \mathrm{mg} / \mathrm{ml}\right.$ in PBS; Sigma-Aldrich; Merck Millipore) at $37^{\circ} \mathrm{C}$ for $3 \mathrm{~h}$, follow by dissolving in DMSO. Finally, the density of cells was detected at $570 \mathrm{~nm}$. Each assay was performed three times and all data presented as the mean \pm standard deviation of the three experiments.

Western blot analysis. The cells were lysed in RIPA buffer containing 1\% NP-40, 50 mM Tris- $\mathrm{HCl}$ (pH 7.4), 1 mM EDTA, $150 \mathrm{mM} \mathrm{NaCl}, 1 \mathrm{mM} \mathrm{NaF}, 0.25 \%$ Na-deoxycholate and $0.2 \mathrm{mM}$ sodium orthovanadate, supplemented with protease inhibitor cocktail. The protein concentration was determined using BCA Protein Assay Reagent (Pierce; Thermo Fisher Scientific, Inc.). Subsequently, $50 \mu \mathrm{g}$ of total protein was subjected to $10 \%$ SDS-PAGE and transferred onto PVDF membranes (EMD Millipore, Billerica, MD, USA). The membranes were then blocked in 5\% skimmed milk at room temperature for $1 \mathrm{~h}$. Subsequently, the membranes were incubated with primary antibodies at $4^{\circ} \mathrm{C}$ overnight, washed with TBST at least three times and then incubated with HRP-conjugated secondary antibodies (1:6,000; cat. nos. ab6721 and ab6789; Abcam, Cambridge, UK) for $2 \mathrm{~h}$ at room temperature. This was followed by washing with TBST three times. The primary antibodies used were as follows: FOXO1 (1:3,000; cat. no. ab39670; Abcam); $\beta$-actin (1:5,000; cat. no. ab8227; Abcam); FLAG (1:5,000; cat. no. F7425; Sigma-Aldrich); EMT antibody sampler kit (1:2,000; cat. no. 9782; Cell Signaling Technology, Inc., Danvers, MA, USA); p16 (1:2,000; cat. no. ab118459; Abcam); p33 (1:3,000; cat. no. ab124893; Abcam); Lsh (1:2,000; cat. no. 7798; Cell Signaling Technology, Inc.); SIRT1 (1:5,000; cat. no. HPA006295; Sigma-Aldrich). $\beta$-actin served as a loading control. Finally, the proteins were visualized using ECL detection reagent (Beyotime Institute of Biotechnology, Dalian, China) according to the manufacturer's protocol. The blots were quantified using Image J software version 1.0 (National Institutes of Health, Bethesda, MD, USA).

RT-qPCR and data analyses. Total cellular RNAs were isolated using TRIzol reagent (Invitrogen; Thermo Fisher Scientific, Inc.), following which a reverse transcription system (Promega A3500; Thermo Fisher Scientific, Inc.) was used for the synthesis of first strand cDNA. Quantitation of the indicated gene transcripts was performed using qPCR. The mRNA level of GAPDH was used as the internal control. A total of $1 \mu \mathrm{l}$ cDNA sample, $1.5 \mu \mathrm{l}$ primer $(10 \mathrm{nM}), 7.5 \mu 1$ 2X MIX buffer and $5 \mu \mathrm{l}$ RNase-free water were added. The qPCR conditions were as follows: $5 \mathrm{~min}$ at $95^{\circ} \mathrm{C}$, denaturation at $95^{\circ} \mathrm{C}$ for $30 \mathrm{sec}$, annealing at $57^{\circ} \mathrm{C}$ for $30 \mathrm{sec}$ and extension at $72^{\circ} \mathrm{C}$ for $40 \mathrm{sec}$, performed for 30 cycles. The primer pairs used were as follows: E-cadherin, forward 5'-AATCTC AAGCTCATGGATAACC-3' and reverse 5'-GCAGAATCA GAATTAGCAAAGC-3'; $\alpha$-catenin, forward 5'-GCTGAA AGTTGTGGAAGATGG-3' and reverse 5'-TTATAGGCT GCGACATCAGG-3'; N-cadherin, forward 5'-TCAAAGCCT GGAACATATGTG-39 and reverse 5'-TGTTTGAAAGGC CATATGTGG-3'; fibronectin, forward 5'-GAGTAAACC TGAAGCTGAAGAG-3' and reverse 5'-TCACCAATCTTG TAGGACTG-3'; GAPDH, forward 5'-ATGAGAAGTATG ACAACAGCCT-3' and reverse 5'-ATGGACTGTGGTCAT GAGTC-3'; and SIRT1, forward 5'-GCACTAATTCCAAGT TCCATACC-3' and reverse 5'-GCAAAGTTTGGCATATTC ACC-3'. Relative mRNA expression was calculated by the $2^{-\Delta \Delta C \mathrm{C}}$ method (22).

Luciferase activity assay. The cells were seeded at $5 \times 10^{4}$ in 6 -well plates, followed by transfection with plasmids when the cells reached $70-80 \%$ confluence. The activities of luciferase following $\sim 48 \mathrm{~h}$ transfection were quantified using an illuminometer (Centro LB 960; Berthold Technologies GmbH, Bad Wildbad, Germany). Each experiment was performed at least three times.

ChIP assay. A ChIP assay was performed using an EZ-ChIP kit (EMD Millipore) according to the manufacturer's protocol with minor modifications. In brief, the LN18 cells 
Table I. Clinicopathologic variables in 143 patients with glioblastoma.

FOXO1 expression

\begin{tabular}{|c|c|c|c|c|}
\hline Variable & No. $(n=143)$ & Low $(n=90)$ & High $(n=53)$ & P-value \\
\hline \multicolumn{5}{|l|}{ Gender } \\
\hline Male & 85 & 56 & 29 & 0.377 \\
\hline Female & 58 & 34 & 24 & \\
\hline \multicolumn{5}{|l|}{ Age (years) } \\
\hline$<50$ & 72 & 45 & 27 & 0.913 \\
\hline$\geq 50$ & 71 & 45 & 26 & \\
\hline \multicolumn{5}{|l|}{ Tumor diameter } \\
\hline Large $(\geq 2 \mathrm{~cm})$ & 61 & 47 & 14 & 0.003 \\
\hline Small $(<2 \mathrm{~cm})$ & 82 & 43 & & \\
\hline \multicolumn{5}{|c|}{ Pathological grade } \\
\hline $\mathrm{I}-\mathrm{II}$ & 70 & 33 & & $<0.001$ \\
\hline III-IV & 73 & 57 & & \\
\hline \multicolumn{5}{|c|}{ Neck lymph node metastasis } \\
\hline No & 69 & & & 0.01 \\
\hline Yes & 74 & & & \\
\hline \multicolumn{5}{|l|}{ Differentiation } \\
\hline Well/moderate & 70 & 49 & 21 & 0.087 \\
\hline Poor & 73 & 41 & 32 & \\
\hline
\end{tabular}

$\left(\sim 3 \times 10^{4}\right)$ were used at a density of $80-90 \%$ confluence, and human astrocytes. The results demonstrated that the expression ChIP assays were performed using $5 \mu \mathrm{l}$ anti-FOXO1 anti- of FOXO1 was decreased at the protein and mRNA levels in body (cat. no. ab39670; Abcam) at 4 at 44ntibod. The DNA these GBM cell lines, compared with the normal human astrowas isolated from the immunoprecipitates and quantified cytes (Fig. 1A). In addition, RT-qPCR analysis was performed using RT-qPCR analysis with the specific primer pairs: to examine the mRNA levels of FOXO1 in seven paired human p16, forward 5'-CTCTTATACCAGGCAATGTA-3'; and GBM tissues and adjacent normal tissues. Similar results reverse 5'-GTACGACTAGAAAGTGTCCC-3'; SIRT1, were obtained, which showed the expression of FOXO1 was forward 5'-CAGATGGATTTCAGAGGGAT-3' and reverse significantly downregulated in the GBM tissues, compared 5'-GAAGGCTGAGGCAGGAGAAT-3'. A' total of $2 \mu \mathrm{l}$ sample, with that in the adjacent normal tissues (Fig. 1B). Together, $1 \mu \mathrm{l}$ primer $(10 \mathrm{nM}), 10 \mu \mathrm{l} 2 \mathrm{X}$ MIX buffer and $7 \mu 1 \mathrm{RNase}$-free these results showed that FOXO1 was downregulated in GBM water were added. The $\mathrm{qPCR}$ conditions were as follows: cell lines and primary tumors. Subsequently, the present study $5 \mathrm{~min}$ at $95^{\circ} \mathrm{C}$, denaturation at $95^{\circ} \mathrm{C}$ for $30 \mathrm{sec}$, annealing at investigated the expression levels of FOXO1 in 143 patients $57^{\circ} \mathrm{C}$ for $30 \mathrm{sec}$ and extension at $72^{\circ} \mathrm{C}$ for $40 \mathrm{sec}$, performed with GBM. The RT-qPCR analysis revealed that the expresfor 30 cycles. Input DNA and DNA immunoprecipitated by anti-IgG served as a positive and negative control, respectively.

Statistical analysis. All results were analyzed using SPSS v. 18.0 (SPSS, Inc., Chicago, IL, USA) and reported as the mean \pm standard deviation. A paired samples t-test was used to compare between adjacent normal tissue and cancer tissues. $\mathrm{P}<0.05$ was considered to indicate a statistically significant difference.

\section{Results}

FOXO1 is downregulated in human GBM cell lines and tissues. In order to examine the function of FOXO1 in GBM in the present study, the expression levels of FOXO1 were determined in the LN18 and T98G GBM cell lines, and normal sion of FOXO1 was significantly downregulated in GBM. The expression of FOXO1 was negatively correlated with pathological grade $(\mathrm{P}<0.001)$, tumor size $(\mathrm{P}=0.003)$ and neck lymph node metastasis $(\mathrm{P}=0.01)$. However, differentiation, age and gender were not correlated with FOXO1 (Table I). In addition, the present study investigated prognosis in the downregulation of FOXO1 in GBM. As expected, the survival curve indicated that the survival rates of patients with a high expression of FOXO1 were significantly higher, compared with those with low expression levels of FOXO1 (hazard ratio=1.49; $\mathrm{P}=0.024$; Fig. 1C).

FOXO1 inhibits GBM cell proliferation. To further examine the role of FOXO1 in GBM, FLAG-FOXO1 was overexpressed in GBM cell lines, LN18 and T98G cells. RT-qPCR and western blot analyses were used to detect the transfection efficiency 
A

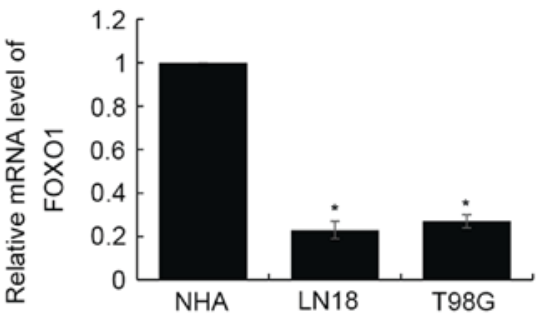

B
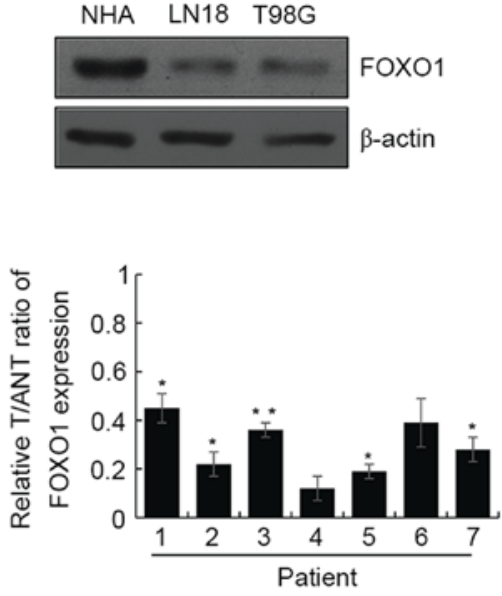

C

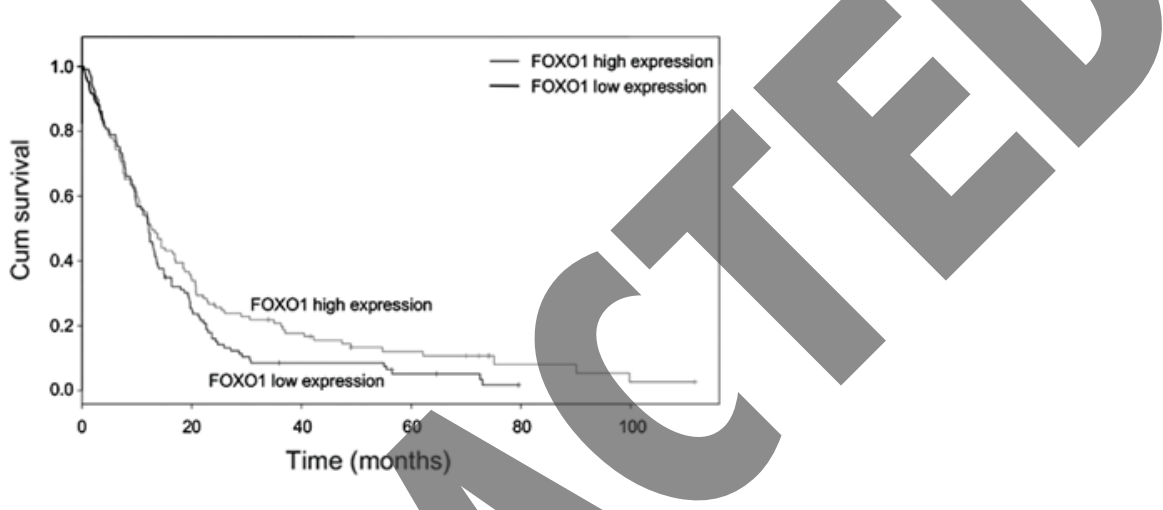

Figure 1. Expression of FOXO1 is downregulated in human GBM cell lines and tissues. (A) Western blot (left) and RT-qPCR (right) analyses of the expression of FOXO1 in NHAs and GBM cell lines, LN18 and T98G. (B) RT-qPCR analysis was used to examine expression levels of FOXO1 in seven paired human GBM tissues and adjacent normal brain tissues, which were collected from patients with GBM. All experiments were repeated three times and data are presented as the mean \pm standard deviation of the three experiments, ${ }^{*} \mathrm{P}<0.05 ;{ }^{*} \mathrm{P}<0.01$. (C) Analysis of the association between FOXO1 and survival in GBM using a Kaplan-Meier curve. GBM, glioblastoma, NHAs, normal human astrocytes; FOXO1, Forkhead Box O1; T, GBM tissue; ANT, adjacent normal tissue; RT-qPCR, reverse transcription-quantitative polymerase chain reaction; Cum, cumulative.

(Fig. 2A and B). Colony formation and MTT assays were also performed to investigate the function of FOXO1 in cell growth in GBM. The colony formation and MTT assays revealed that FOXO1 significantly decreased colony forming ability and cell growth in the LN18 and T98G cells (Fig. 2C and D). FOXO1 siRNA was also used to knock down FOXO1 in the LN18 and T98G cells, followed by analysis using colony formation and MTT assays. The depletion of FOXO1 increased the colony forming ability in cell proliferation of the LN18 and T98G cells (Fig. 2E). To examine whether FOXO1 affected cell cycle progression, flow cytometry was used to detected cell cycle. The results revealed that the overexpression of FOXO1 arrested the cell cycle in the $G_{0} / G_{1}$ phase, suppressing cells from entering the $\mathrm{S}$ phase (Fig. $2 \mathrm{~F}$ ). By contrast, FOXO1 knockdown resulted in a higher number of cells entering the $S$ phase, follow by promoted cell growth (Fig. 2G). In brief, these findings demonstrated that FOXO1 arrested cells at the $\mathrm{G}_{0} / \mathrm{G}_{1}$ phase and suppressed cell growth in GBM.

FOXO1 promotes senescence via the transcriptional inhibition of SIRT1. Following the induced overexpression of FOXO1, p16 ${ }^{\text {INK4a }}$ was also expressed at a high level (Fig. 3A). p16 ${ }^{\text {INK4a }}$ has been reported to be expressed at high levels in cell senescence, in which it has a crucial function. Therefore, it was hypothesized that FOXO1 may be involved in cell senescence of GBM. There are several proteins associated with cell senescence, including histone deacetylase (HDAC)4, SIRT1, lymphoid specific helicase (Lsh) and p33 ${ }^{\text {ING1b }}$. To confirm this hypothesis, the present study investigated whether FOXO1 regulated these proteins. As shown in Fig. 3A, in LN18 cells overexpressing FOXO1, the expression of SIRT1 was suppressed, whereas HDAC4, Lsh and p33 $3^{\text {ING1b }}$ were not affected. The opposite results were obtained in the FOXO1-depleted cells, which showed SIRT1 was increased and p16 ${ }^{\text {INK4a }}$ was decreased (Fig. 3B). The mRNA levels of SIRT1 and $\mathrm{p} 16^{\mathrm{INK} 4 \mathrm{a}}$ were also regulated by FOXO1, which suggested that FOXO1 regulated SIRT1 and $\mathrm{p} 16^{\mathrm{INK} 4 \mathrm{a}}$ at the transcriptional level (Fig. 3C). The present study then examined whether FOXO1 affected the transcription $16^{\text {INK4a }}$ or SIRT1. A ChIP assay was performed with FOXO1 antibody, which revealed that the SIRT1 promoter region interacted with FOXO1, whereas $16^{\mathrm{IN} 4 \mathrm{a}}$ did not interact with FOXO1. Although the mRNA and protein levels of p16 ${ }^{\mathrm{IN} 4 \mathrm{a}}$ were affected by FOXO1, FOXO1 did not interact with its promoter region, therefore, FOXO1 may be indirectly regulated $\mathrm{p} 16^{\mathrm{IN} 4 \mathrm{a}}$ by other proteins (Fig. 3D). The luciferase reporter assay also confirmed that the recruitment of wide-type FOXO1 (wt-FOXO1) interacted with the SIRT1 promoter region and negatively regulated its 


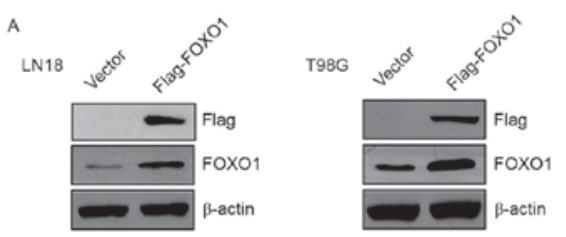

B

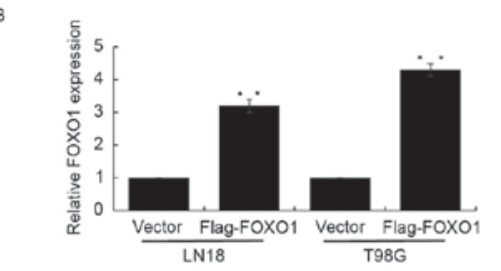

C
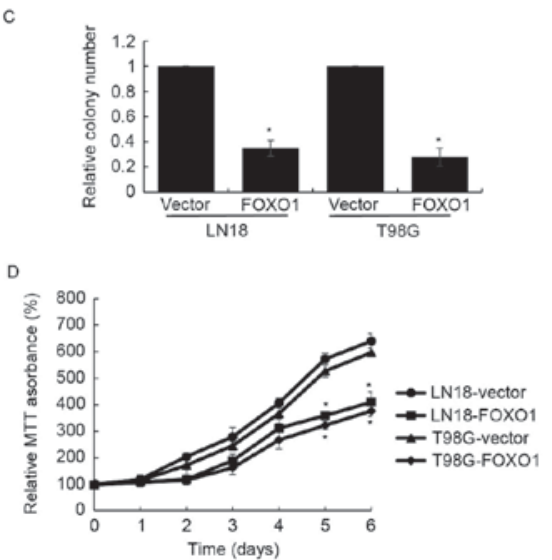

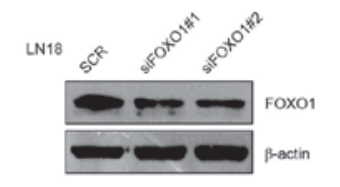

T986
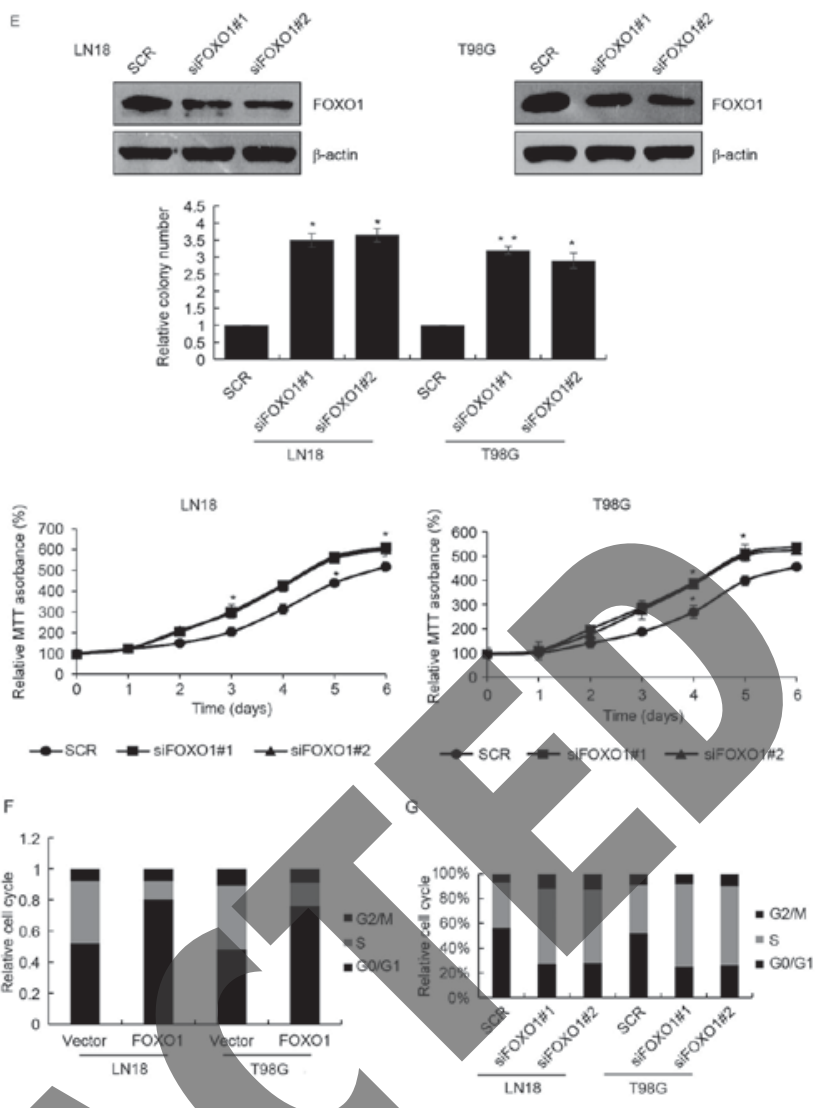

Figure 2. FOXO1 inhibits glioblastoma cell proliferation. (A) Validation of expression levels of FOXO1 following transfection in LN18 (left) and T98G (right) cells by western blot analysis. (B) Validation of expression levels of FOXO1 following transfection in LN18 (left) and T98G (right) cells by reverse transcription-quantitative polymerase chain reaction analysis." $\mathrm{P}<0.01$. (C) FOXO1 was overexpressed in LN18 and T98G cells and cell colonies were counted. ${ }^{*} \mathrm{P}<0.05$. (D) MTT assays demonstrated that that overexpression of FOXO1 significantly inhibited growth of the LN18 and T98G cells. " $\mathrm{P}<0.05$. (E) Efficiency of FOXO1 siRNA in LN18 or T98G cells was verified using western blot analysis. FOXO1 was knocked down in LN18 and T98G cells, and colony formation and MTT assays were performed. ${ }^{*} \mathrm{P}<0.05,{ }^{* *} \mathrm{P}<0.01$. (F) Flow cytometric analysis revealed LN18 and T98G cells transfected with the vector or FOXO1. Data are presented as the mean \pm standard deviation of the three experiments. (G) Flow cytometric analysis revealed LN18 and T98G cells transfected with FOXO1 siRNA. Each bar indicates the mean of triplicate independent experiments. FOXO1, Forkhead Box O1; siRNA, small interfering RNA.

transcription. However, FOXO1 (K245A) failed to regulate the transcription of SIRT1 (Fig. 3E)

FOXO1 inhibits epithelial-mesenchymal transition (EMT) and metastasis in GBM. To determine the role of FOXO1 in the EMT of GBM cells, the present study examined the expression of EMT markers in LN18 cells with FOXO1 depletion. The results showed that, at the mRNA and protein levels, the expression of epithelial markers, E-cadherin and $\alpha$-catenin were decreased, and the expression of mesenchymal markers, $\mathrm{N}$-cadherin, fibronectin and vimentin were increased (Fig. 4A). The opposite results were observed in cells overexpressing FOXO1, where the expression of epithelial markers E-cadherin and $\alpha$-catenin were significantly increased, and expression of mesenchymal markers $\mathrm{N}$-cadherin, fibronectin and vimentin were decreased (Fig. 4B). These results indicated that FOXO1 regulated EMT programming in the GBM cells. There are several reports indicating that EMT promotes cell invasion. In the present study, it was found that FOXO1 was negatively correlated with lymph node metastasis, therefore, FOXO1 may suppress GBM cell invasion. Transwell assays were performed to confirm this hypothesis; in the highly invasive human U87 GBM cancer cell line, the results indicated that, following knockdown of FOXO1, the number of invaded cells through Matrigel was almost three times as high as in the control, which revealed that the invasion ability of the U87 cells was enhanced when FOXO1 was knocked down (Fig. 4C). By contrast, when FOXO1 was overexpressed, the number of invaded cells was decreased, compared with the number in the control group (Fig. 4C). Therefore, FOXO1 affected the invasion ability of U87 cells. The present study also investigated the role of FOXO1 in anchorage-independent cell growth. As shown in Fig. 4D, the knockdown of FOXO1 in U87 cells significantly promoted colony formation. Therefore, FOXO1 has a key function in several cancer development processes, including EMT and cell invasion, and it also suppresses the anchorage-independent grow ability of cancer cell.

\section{Discussion}

In the present study, it was found that FOXO1 was significantly downregulated in GBM primary tumors and cell lines. The expression of FOXO1 was negatively correlated with clinical pathology, including neck lymph node metastasis $(\mathrm{P}=0.01)$ and tumor size $(\mathrm{P}=0.003)$. The $\mathrm{K}-\mathrm{M}$ plot analysis survival curve also indicated that a high expression of FOXO1 in GBM was 
A

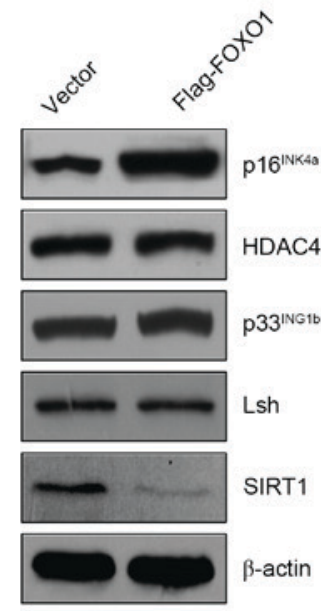

C

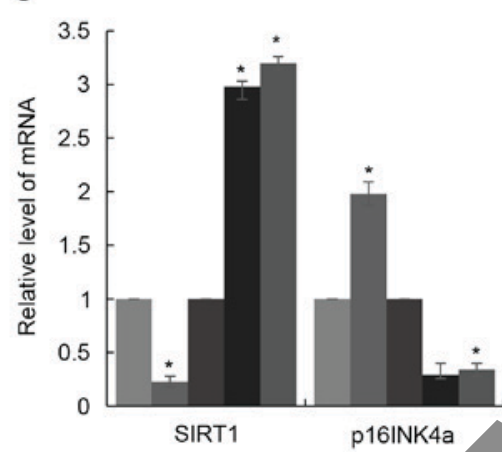

B

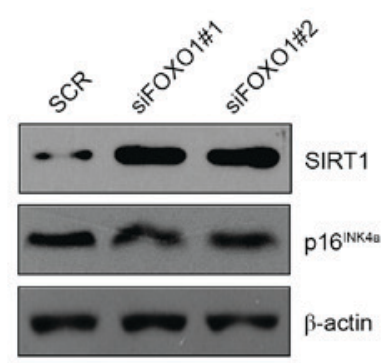

E

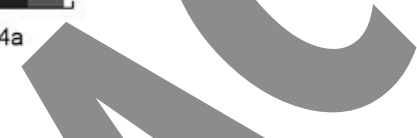

D
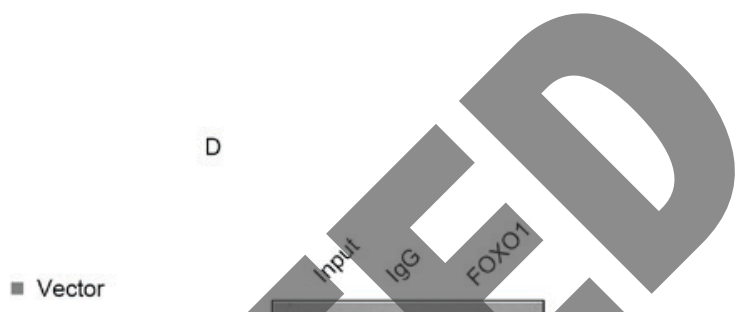

- Flag-FOX01

- SCR

- siFOX01\#1

- siFOX01\#2

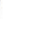
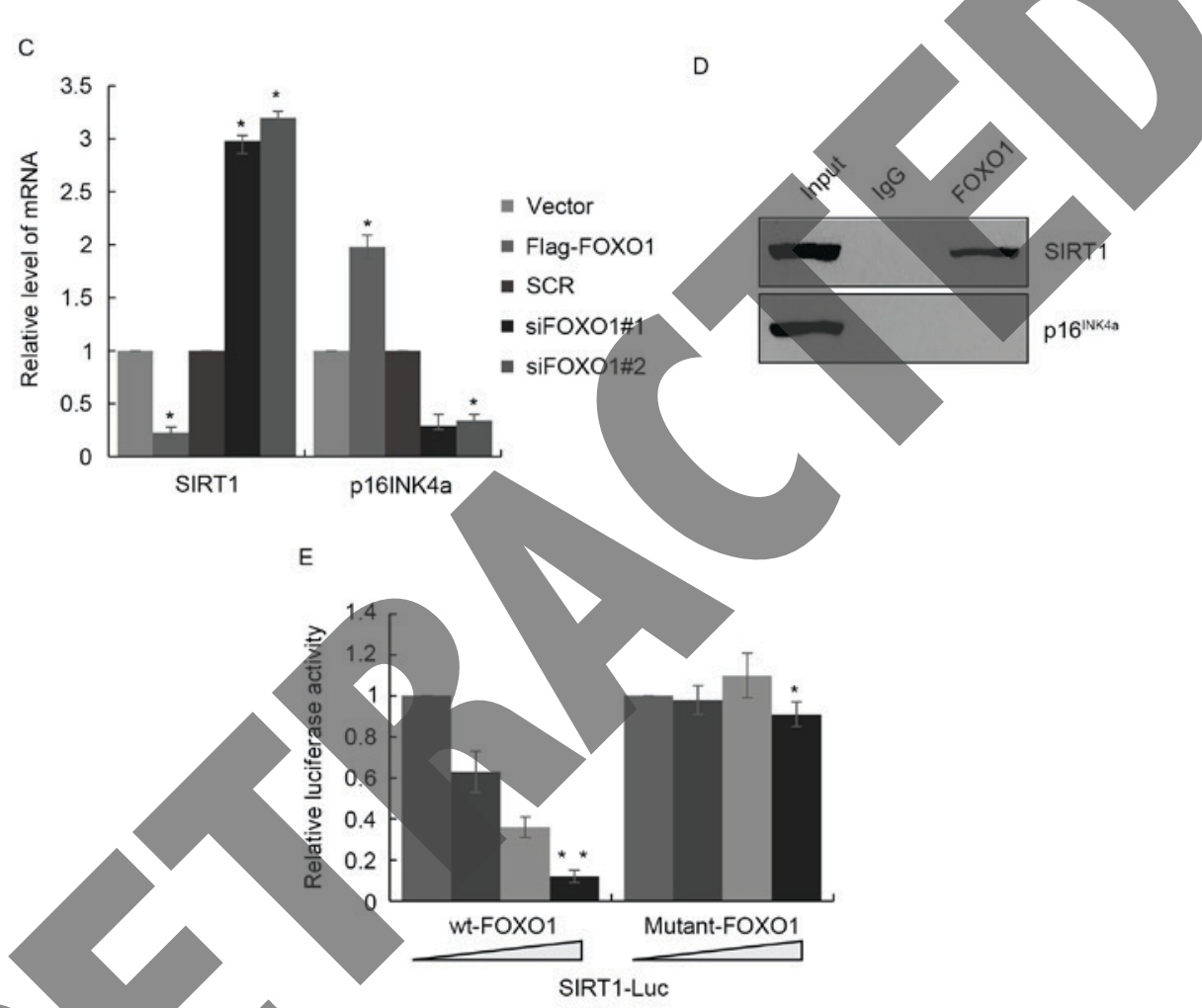

Figure 3. FOXO1 promotes senescence via the transcriptional inhibition of SIRT1. (A) Validation of marker of senescence of p16 ${ }^{\text {INK4a }}$, HDAC4, p33 ${ }^{\text {INK1b }}$, Lsh and SIRT1 in LN18 cells overexpressong FOXO1. (B) Knockdown of FOXO1 by FOXO1 siRNA in LN18 cells, followed by the detection of expression levels of SIRT1 and p16 ${ }^{\text {INK4a }}$. (C) Reverse transcription-quantitative polymerase chain reaction analysis revealed mRNA levels of SIRT1 and p16 $6^{\text {INK4a }}$ were altered in LN18 cells overexpressing FOXO1 or in those with FOXO1 knockdown. ${ }^{*} \mathrm{P}<0.05$. (D) ChIP assay in LN18 cells using anti-FOXO1 antibody. (E) Luciferase reporter assays in LN18 cells co-transfected with SIRT1-Luc and wt FOXO1 or FOXO1 (K245A) as indicated. Data are presented as the mean \pm standard deviation of the three experiments. "P $<0.05$; $\mathrm{P}<0.01$. FOXO1, Forkhead Box O1; SIRT1, sirtuin 1; siRNA, small interfering RNA; HDAC4, histone deacetylase 4; Lsh, lymphoid specific helicase; wt, wild-type.

positively correlated with increased survival rates. Colony formation and MTT assays revealed that, following the ectopic expression of FOXO1, FOXO1 suppressed GBM cell growth. When FOXO1 siRNA was used to knock down FOXO1 in the GBM cells, the opposite results were obtained. The present study also found that the overexpression of FOXO1 arrested GBM cells at the $\mathrm{G}_{0} / \mathrm{G}_{1}$ phase and inhibited cell proliferation, whereas FOXO1 knockdown released cells at the $\mathrm{G}_{0} / \mathrm{G}_{1}$ phase for entry into the $\mathrm{S}$ phase.

The present study also found that the ectopic expression of FOXO1 resulted in p16 $6^{\mathrm{INK} 4 \mathrm{a}}$ being expressed at a high level. The tumor suppressor $\mathrm{p} 16^{\mathrm{INK1a}}$ has been reported to be crucial in cell senescence (23). Various types of stress result in decreased cell proliferation, following regulation by tumor suppressor genes, including p53, p16 $6^{\text {INK4a }}$ and p $21(23,24)$. Several proteins have been found to be involved in cell senescence, including HDAC4, SIRT1, p33 ${ }^{\text {ING1b }}$ and LSH (25-27). The present study investigated whether FOXO1 affected the expression of these genes. The results showed that FOXO1 regulated the expression of SIRT1, but had no effect on the expression of HDAC, LSH or $\mathrm{p} 33^{\text {ING1b}}$. The ChIP assay demonstrated that FOXO1 bound to the promoter region of SIRT1 in GBM cells, and decreased the mRNA and protein levels of SIRT1 in GBM cells. These results suggested that FOXO1 decreased the expression of 
A

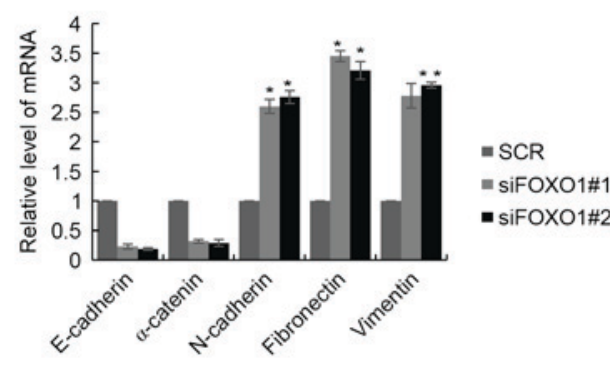

B

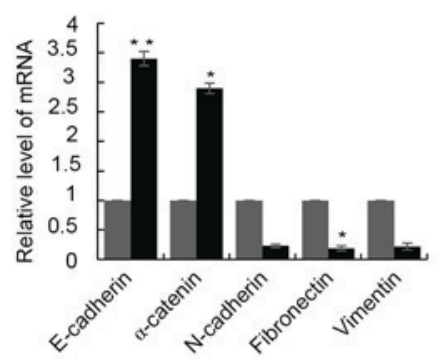

C

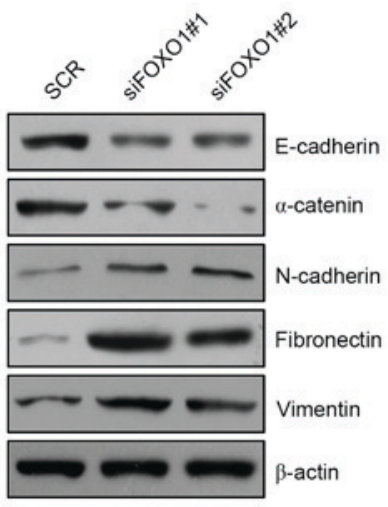

$=$ Vector

- Flag-FOXO1

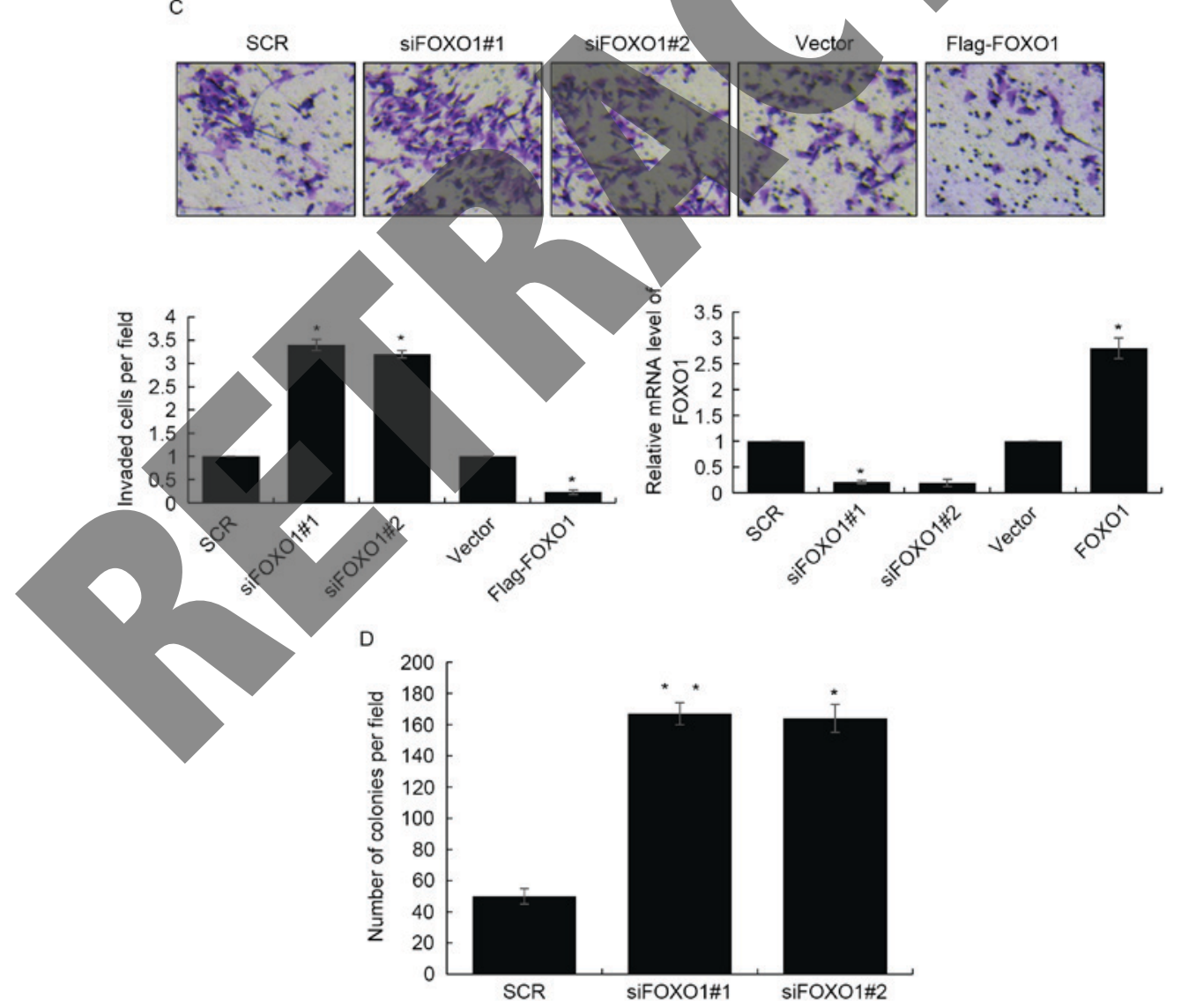

Figure 4. FOXO1 inhibits EMT and metastasis in glioblastoma. (A) RT-qPCR (left) and western blot (right) analyses detected mRNA and protein levels of EMT markers in LN18 cells with FOXO1 knockdown. "P<0.05. (B) RT-qPCR (left) and western blot (right) analyses detected mRNA and protein levels of EMT markers in LN18 cells overexpressing FOXO1. "P<0.05; ${ }^{* *} \mathrm{P}<0.01$. (C) For cell invasion assays, U87 cells were transfected with the FOXO1 siRNAs or expression constructs. The efficiency of siRNA and plasmid were detected using RT-qPCR analysis. Cells were stained with crystal violet and invaded cells were counted (mangification, $x 40$ ). Each bar indicates the mean of triplicate independent experiments. "P<0.05. (D) U87 cells were transfected with FOXO1 siRNA and a soft agar colony formation assay was performed. Data are presented as the mean + standard deviation of the three experiments. ${ }^{*} \mathrm{P}<0.05 ;{ }^{* * *} \mathrm{P}<0.01$. EMT, epithelial-mesenchymal transition; FOXO1, Forkhead Box O1; RT-qPCR, reverse transcription-quantitative polymerase chain reaction; siRNA, small interfering RNA; SCR, scramble siRNA. 
SIRT1 at the transcriptional level and that FOXO1 promoted senescence via the transcriptional inhibition of SIRT1.

The data obtained in the present study demonstrated that FOXO1 inhibited EMT. EMT is a complex process in cancer cell development, which involves cancer cell growth, different, metastasis and other cell processes. The ectopic expression of FOXO1 markedly increased epithelial markers, including E-cadherin and $\alpha$-catenin, whereas mesenchymal markers, $\mathrm{N}$-cadherin, fibronectin and vimentin were all decreased. The opposite results were obtained when FOXO1 was silenced. Metastasis is a characteristic of malignant cancer and results in poor prognosis. EMT enhances the metastasis of cancer cells. As the present study found that FOXO1 was negatively correlated with neck lymph node metastasis, whether FOXO1 regulated cancer metastasis was determined using a Transwell assay. The results revealed FOXO1 suppressed GBM cell metastasis.

Taken together, the results of the present study revealed that FOXO1 was downregulated in GBM and, as a tumor suppressor, FOXO1 inhibited tumor growth and senescence via arresting cell cycle at the G0/G1 phase and inhibiting the transcription of SIRT1, respectively. FOXO1 also suppressed EMT and metastasis. These results provide evidence that FOXO1 may be a potential biomarker and therapeutic target for patients with GBM.

\section{References}

1. Sullivan PR: Brain tumors. N Engl J Med 344: 1478, 2001.

2. Fu Z and Tindall DJ: FOXOs, cancer and regulation of apoptosis. Oncogene 27: 2312-2319, 2008.

3. Paik JH, Kollipara R, Chu G, Ji H, Xiao Y, Ding Z, Miao L Tothova Z, Horner JW, Carrasco DR, et al: FoxOs are lineage-restricted redundant tumor suppressors and regulate endothelial cell homeostasis. Cell 128:309-323, 2007.

4. Sunayama J, Sato A, Matsuda K, Tachibana K, Watanabe E, Seino S, Suzuki K, Narita Y, Shibui S, Sakurada K, et al: FoxO3a functions as a key integrator of cellular signals that control glioblastoma stem-like cell differentiation and tumorigenicity. Stem Cells 29: 1327-1337, 2011.

5. Morin RD, Mendez-Lago M, Mungall AJ, Goya R, Mungall KL, Corbett RD, Johnson NA, Severson TM, Chiu R, Field M, et al: Frequent mutation of histone-modifying genes in non-Hodgkin lymphoma. Nature 476: 298-303, 2011.

6. Trinh DL, Scott DW, Morin RD, Mendez-Lago M, An J, Jones SJ, Mungall AJ, Zhao Y, Schein J, Steidl C, et al: Analysis of FOXO1 mutations in diffuse large B-cell lymphoma. Blood 121 3666-3674, 2013

7. Huang $\mathrm{H}$ and Tindall DJ: Dynamic FoxO transcription factors. J Cell Sci 120: 2479-2487, 2007.

8. Furuyama T, Nakazawa T, Nakano I and Mori N: Identification of the differential distribution patterns of mRNAs and consensus binding sequences for mouse DAF-16 homologues. Biochem J 349: 629-634, 2000.

9. Gilley J, Coffer PJ and Ham J: FOXO transcription factors directly activate bim gene expression and promote apoptosis in sympathetic neurons. J Cell Biol 162: 613-622, 2003.
10. Ishikura S, Iwaihara Y, Tanaka Y, Luo H, Nishi K, Doi K, Koyanagi M, Okamura T, Tsunoda T and Shirasawa S: The nuclear zinc finger protein Zfat maintains FoxO1 protein levels in peripheral $\mathrm{T}$ cells by regulating the activities of autophagy and the Akt signaling pathway. J Biol Chem 291: 15282-15291, 2016.

11. Wang S, Xia P, Huang G, Zhu P, Liu J, Ye B, Du Y and Fan Z: FoxO1-mediated autophagy is required for NK cell development and innate immunity. Nat Commun 7: 11023, 2016

12. Milan G, Romanello V, Pescatore F, Armani A, Paik JH, Frasson L, Seydel A, Zhao J, Abraham R, Goldberg AL, et al: Regulation of autophagy and the ubiquitin-proteasome system by the FoxO transcriptional network during muscle atrophy. Nat Commun 6: 6670, 2015.

13. Zhao Y, Yang J, Liao W, Liu X, Zhang H, Wang S, Wang D, Feng J, Yu L and Zhu WG: Cytosolic FoxO1 is essential for the induction of autophagy and tumour suppressor activity. Nat Cell Biol 12: 665-675, 2010.

14. Guarente L and Picard F: Calorie restriction-the SIR2 connection. Cell 120: 473-482, 2005 .

15. Longo VD and Kennedy BK: Sirtuins in aging and age-related disease. Cell 126: 257-268

16. Li X, Zhang S, Blander G, Tse JG, Krieger M and Guarente L: SIRT1 deacetylates and positively regulates the nuclear receptor LXR. Mol Cell 28: 91-106, 2007.

17. Lemieux ME, Yang $X$, Jardine $K$, He $X$, Jacobsen $K X$, Staines WA, Harper ME and McBurney MW: The Sirt1 deacetylase modulates the insulin-like growth factor signaling pathway in mammals. Mech Ageing Dev 126: 1097-1105, 2005.

18. Ramsey KM, Mills KF, Satoh A and Imai S: Age-associated loss of Sirt1-mediated enhancement of glucose-stimulated insulin secretion in beta cell-specific Sirt1-overexpressing (BESTO) mice. Aging Cell 7: 78-88, 2008.

9. Paroni G, Mizzau M, Henderson C, Del Sal G, Schneider C and Brancolini C: Caspase-dependent regulation of histone deacetylase 4 nuclear-cytoplasmic shuttling promotes apoptosis. Mol Biol Cell 15: 2804-2818, 2004.

20. Paroni G, Fontanini A, Cernotta N, Foti C, Gupta MP, Yang XJ, Fasino D and Brancolini C: Dephosphorylation and caspase processing generate distinct nuclear pools of histone deacetylase 4. Mol Cell Biol 27: 6718-6732, 2007.

1. Dole MG, Jasty R, Cooper MJ, Thompson CB, Nunez G and Castle VP: Bcl-xL is expressed in neuroblastoma cells and modulates chemotherapy-induced apoptosis. Cancer Res 55: 2576-2582, 1995.

22. Livak KJ and Schmittgen TD: Analysis of relative gene expression data using real-time quantitative PCR and the 2(-Delta Delta C(T)) method. Methods 25: 402-408, 2001

23. Lundberg AS, Hahn WC, Gupta P and Weinberg RA: Genes involved in senescence and immortalization. Curr Opin Cell Biol 12: 705-709, 2000.

24. Campisi J: Cellular senescence as a tumor-suppressor mechanism. Trends Cell Biol 11: S27-S31, 2001.

25. Zhou R, Han L, Li G and Tong T: Senescence delay and repression of p16INK4a by Lsh via recruitment of histone deacetylases in human diploid fibroblasts. Nucleic Acids Res 37: 5183-5196, 2009.

26. Han X, Niu J, Zhao Y, Kong Q, Tong T and Han L: HDAC4 stabilizes SIRT1 via sumoylation SIRT1 to delay cellular senescence. Clin Exp Pharmacol Physiol 43: 41-46, 2016.

27. Li N, Li Q, Cao X, Zhao G, Xue L and Tong T: The tumor suppressor p33ING1b upregulates p16INK4a expression and induces cellular senescence. FEBS Lett 585: 3106-3112, 2011. 